\title{
Zooming Through Covid: Fostering Safe Communities of Critical Reflection via Online Writers' Group Interaction
}

\author{
Sarah Haas, Alexander De Soete, and Gry Ulstein \\ Ghent University
}

\section{Introduction}

Writers' groups are nothing new (Gere, 1987), and the benefits of writing as a social activity, whether in writers' groups or at writing retreats, are well-established in many disciplines (e.g., increased output, fewer feelings of isolation, and better written product [Aitchison \& Guerin, 2014; Elbow, 1998; Kornhaber et al., 2016; Murray, 2015]). With the advent of digital tools allowing reliable remote communication, online writers' groups and retreats are becoming a commonplace complement to face-to-face social writing spaces ( 0 'Dwyer et al., 2017). Writers' groups have long been conceptualized as "communities of practice," which have been found to, among other things, provide emotional safety for community members (Badenhorst et al., 2019; Thesen, 2014). The emotional safety found in communities of practice that meet in physical spaces has been found to transfer to virtual communities as well (Hennekam et al., 2019; Ji, et al. 2017). The Covid-19 pandemic, bringing with it the necessity of working from home and social distancing, has thrown into sharp relief the potential benefits of online writers' groups. In this report from the field, we suggest that it is precisely the safety that is fostered in virtual communities of writers that opens a space for critical thinking-more specifically, critical reflection on writing processes-which helps writers manage and optimise their processes in a supportive community-of-practice setting, even in less-than-ideal circumstances.

Critical thinking and reflective thinking are generally agreed upon as essential higherorder thinking skills-ones we want to cultivate in our education programs (Ghanizadeh, 2017). What seem to be open for discussion in the literature, however, are exact definitions and agreed-upon understandings regarding the relationship between the two (Erdogan, 2020; Ghanizadeh, 2017; Rademaekers \& Detweiler, 2019). While a full examination of definitions and of the chicken-egg relationship between critical thinking and reflective thinking are beyond the scope of this report, we take the stance that some kinds of reflection, specifically critical reflection, cannot take place unless there is first a solid foundation of critical thinking. Kember et al. (2008) situated "critical reflection" as one of four types of reflection: a "deeper kind" that exmines underlying beliefs and habits (Kember et al. 2008, p. 374 ), that "develops the awareness ... of the [practitioner-in this case writer] of the practices in the process ... and triggers the [practitioners/writers] to make positive changes in [their] practices" (Erdogan, 2020, p. 232). Thus, we presume that critical reflectionwhether done on an indivicual basis or via group discussions-is firmly rooted in critical thinking.

The goal of this report is to discuss the merits we observed in our online writers' groups with regard to community-building, safety, and critical reflection, and by doing so, to encourage the organization of online writers' groups in similar contexts. 


\section{Situation}

On Friday March 13, 2020, on-campus teaching at Ghent University was stopped, disrupting the status quo and causing uncertainty and anxiety among staff and students alike. Following the first lockdown measures, our undergraduate students reported to teaching staff feelings of "disorientation" by the abrupt change and "feeling lost" by the lack of structure in their days-particularly by suddenly having no opportunities for the customary face-to-face support from teachers and fellow students. This anxiety and disorientation were evident among $3^{\text {rd }}$-year students enrolled in an English Linguistics seminar (hereafter "LSem") taught by A. De Soete. The LSem students were working on research projects for which they had to write 10,000-word reports. The course had provided support and guidance in research and writing processes by means of weekly classes. However, even after moving these classes online (using Zoom as videoconferencing and teaching platform), De Soete still perceived a need for additional support for the students.

De Soete and G. Ulstein had recently attended a Writer Development course for PhD students (Haas, 2020), where they had been introduced to the benefits of social writing models, such as the typing pool (Murray, 2015) and Shut Up and Write (Mewburn et al., 2014). De Soete decided to implement extra support for the LSem students by organizing regular online writers' groups. The remainder of this report provides an account of the organization and implementation of this LSem writers' group. We use existing theory on writers' groups to support observations made during 23 recorded writing sessions and one feedback-and-reflection session organized at the end of the semester by Haas, De Soete, and Ulstein (henceforth referred to as the "facilitators"). All students provided informed consent to having the sessions recorded and used for research purposes.

\section{Basic Set-Up of the Intervention}

The supplementary, non-compulsory writers' group meetings were presented to the LSem students as a community of writers all needing to get work done. The meetings ran from April 6 to June 25 and were organized twice a week: on Mondays from 10:00 am to 1:00 pm and Thursdays from 2:30 to 5:30 pm, with the facilitators sending out online meeting invitations (via Zoom) before each meeting. There was a "floating membership" (Haas, 2014, p. 34), with people showing up for the meeting if and when they wanted to. There were 20 students enrolled in the course; in the writing sessions, there was always a minimum of six participants and at most eighteen. The facilitators served as "start-up leaders" (Haas, 2014, p. 39) but quickly began to rotate the "host" role among the students.

\section{The Semantics of a Flat Hierarchy}

The title of "host" was adopted instead of "leader" not only because it is consistent with the role on the Zoom platform (the "host" is Zoom's title for whoever schedules the meeting), but also because it was a priority of the facilitators to keep any sense of hierarchy as flat as possible. We saw this as important to community-building, which was one of the key principles of the intervention, as it conformed to the needs expressed by the students in midMarch. Moreover, we viewed it as a way to reduce anxiety among students who had expressed a reluctance to taking on the role of "leader" because they associated this role with the pressure of having to be an expert. This was confirmed by students in a feedback session at the end of the semester, during which the students reflected that "hosting" as opposed to 
"leading" or "coaching" implies taking turns having friends around and thus serves the purposes of a non-hierarchical structure, which carries fewer "expert" connotations.

Originally, the host duties were based on an implicit playbook by which hosts welcomed the participants, managed the time, and moderated the dialogue, but as will be discussed below, individual hosts started experimenting with more personal twists on the host duties. The rotating host role became a key part of the writers' group structure, not just for facilitating a flat hierarchy, but also for ensuring the group's discipline and accountability.

\section{Flexible Structure, Friendly Discipline}

Structure and discipline are known to be important for the success of writers' groups (Murray \& Newton, 2009). To maintain a clear and consistent structure, the meetings comprised four parts, as illustrated by Figure 1 below (see also Appendix for a more elaborate visual overview of the structure, including instructions for the host). They began with an informal opening, followed by individual goal-setting, and then stuck to a brief-workdebrief-break sequence (Haas \& Kobayashi, 2014), the latter repeated three times. Although this structure became the general modus operandi, there was always room for flexibility and context-specificity, and adjustments could be made depending on the session-by-session needs of the group. For example, if the facilitator or host (or sometimes, the group together) observed elevated levels of stress or anxiety among the attendees, more time was allotted to the brief and debrief parts. The meetings ended with a reminder of the date and time of the next session and a selection of the next host.

Figure 1 Structure of the Online Writing Sessions

\begin{tabular}{|c|c|c|}
\hline Stage & Timing & Sub-stage \\
\hline Opening/Welcome & $+/-5 \min$ & \\
\hline $\begin{array}{l}\text { Individual } \\
\text { Goal-setting }\end{array}$ & $+/-5 \min$ & \\
\hline \multirow{4}{*}{$\begin{array}{l}\text { Working Sessions } \\
\text { (repeated } 3 \text { times) }\end{array}$} & $+/-5 \min$ & Brief \\
\hline & $45-60 \mathrm{~min}$ & Shut up and Work \\
\hline & $+/-5 \min$ & Debrief \\
\hline & $10-15 \mathrm{~min}$ & Break \\
\hline $\begin{array}{l}\text { Planning for Next } \\
\text { Meeting }\end{array}$ & $+/-5 \min$ & \\
\hline
\end{tabular}

While previous research suggests it can be beneficial for writers to use time in writers' groups exclusively for "real writing," meaning actually putting words on paper (Mewburn et al., 2014; Murray, 2015), De Soete had observed in his classes that if the groups were to be of benefit to students, some of them would need to use the sessions for other research-related activities, such as reading, data analysis or transcription. He therefore established that the groups would take a wider view of writing (such as that found in Haas 
[2009]). This view was made clear to participants before the first Zoom session, and in later meetings the group explicitly agreed that most academic work was considered a valid activity for the duration of the sessions. Other adjustments and in-group "rules" developed as the writers' group progressed and participants became more comfortable with shaping the meetings to accommodate the group's needs. The online format provided an environment that presented participants with both opportunities and challenges that differ from on-campus writers' groups.

\section{Online Platform Adaptations}

The Covid-19 lockdown regulations demanded online learning solutions across academia internationally. There is no way of knowing whether the LSem writers' group would have developed and solidified the way it did were it not for this extreme context. However, the context in which everyone had to turn to online formats offered opportunities that might not otherwise have been discovered. The most obvious opportunity is that the online format enables people at different physical locations to gather and interact in one space. As we chose the platform Zoom for our writers' group, this choice had further implications for the organization of the sessions.

\section{Zoom-Specific Adaptations}

Zoom allows for up to one hundred participants to join the same meeting. While this function presented an opportunity for reaching a large group of students, it also meant that the facilitators and hosts needed to make active use of another Zoom function: the "break-out rooms." The break-out room is a feature that allows the Zoom "host" to divide participants into smaller groups for a set amount of time. The break-out rooms typically served as spaces for brief-and-debrief (part 3) before all participants rejoined the main session to shut-upand-work.

Both in the larger and smaller (break-out-room) groups, one challenge was taking turns speaking when the whole group was gathered-especially during the opening (part 1) and planning (part 4). Sometimes the group would try to engage in more naturally flowing conversations, but the online format complicated the process because only one person could really speak intelligibly at once. This obstacle made the role of the host and/or facilitators as moderator(s) more important, especially if participants were experiencing technical difficulties, such as a poor WiFi connection. Here, however, participants demonstrated a great willingness to help moderate each other, making sure everyone who wanted to say something had a turn to speak.

Zoom further allows participants to (un)mute themselves and turn their camera on and off as they please, features that proved handy: during individual goal-setting (part 2) and shut-up-and-work (part 3), it became common practice to mute microphones while working. Moreover, some participants felt more comfortable turning their camera off while working (during shut-up-and-work) while others kept their cameras on for an increased sense of accountability and community spirit. Participants would sometimes turn their cameras off for a while in the middle of a session without this being disruptive.

Some implicit adjustments were made in order to add to the comfort of the hosts: As mentioned above, the role of the hosts was mainly to be timekeepers and moderators during the various steps of the writing session, and some of the first-time hosts chose to stick closely 
to this role. More experienced hosts would, in addition to the "basic" duties, monitor the mood and needs of the group and adjust the procedures accordingly (see also Appendix).

\section{The Brief and Debrief: "Safety” and “Community” as Important Factors for Success}

When the group closed at the end of the academic year, we were overall pleased with how the intervention had turned out: beyond our own professional and personal enjoyment of the process, we noted that most of the regular participants had completed their writing projects within the time span of the writers' group (April to June 2020); the stragglers did eventually finish as well. Moreover, membership in the meetings was not only consistent (no meetings were canceled due to non-attendance), but the group even expanded to include a few stowaways: several of the original members invited fellow students, family members, friends from other disciplines-or other universities - to join in. Thus, in the end, the membership was not limited to LSem undergrads, but included PhD students, a postdoctoral researcher, and an assistant professor, as well as a group of undergraduates from a range of disciplines. Along with the successful output and expanded membership, we were impressed that the students themselves started to regularly organize their own writers' groups alongside the official ones. In the two weeks leading up to the writing deadline, this occurred on an almost daily basis, including on weekends.

Along with our own positive impression of the intervention, we were also interested in gaining the perspective of the students, so we held a general focus group when the writers' group drew to a close. This focus group was, as were all the Zoom sessions, recorded with the explicit permission of the participants. In the conversation, the students foregrounded the themes of "community" and (emotional) "safety" as important factors that contributed to their continued participation. The focus group reported a shared feeling that the group members were not simply present, but also truly supportive of each other, and while the pressures of writing remained high during the online sessions, the group provided a safe haven. While a full analysis of the discourse in the writers' group is beyond the scope of this report from the field, we were curious to know how the interaction in the group might have created the feelings of community and safety that the students found so important. Looking specifically at the recordings of meetings, along with our field notes made during these meetings, we were able to uncover some of the contributing factors.

\section{We're All in This Together}

From the outset there was clear evidence that participants were not in it only for themselves. From meeting to meeting, people kept track of the topics and states of completion of their colleagues' papers. By the third meeting, rather than being a recitation of individual goals in turn, the briefs had already started to become a place of support. Participants actively remembered what others had been doing in previous group meetings (a goal set, a deadline, a particular problem they were having), and checked up on each other regarding their progress or emotional states. Participants' consideration and concern for each other, and not solely for their own progress, very likely contributed to the feeling of community that the students spoke of.

Establishing That "it's okay"

What also stood out was how the group quickly established a culture of "it's okay." In the debrief, where participants reported how the working hour had gone, a precedent was set 
early on by one of the participants, who reported: "I'm a complete failure. I didn't get any of my goals done." This candid statement may have helped kick-start the general vibe in the group where success was not the only result reported in the debrief. The group's reaction to this exposure of perceived failure was one of support and immediate reassurance that "it's okay," which likely sowed the seeds of safety in the group. While the stress and pressures of writing and other academic activities were still there, the knowledge that "in this group, it's okay to fail" lowered the threshold for admitting to failure, and thus probably contributed to safety, community-building, and willingness to critically reflect on the perceived failures. It was okay to be imperfect, and occasionally, if someone needed to have a meltdown, it was also okay to be not-okay.

The "it's okay" culture extended to the brief as well, where the bold reporting of potential pitfalls due to personal distractions no doubt also contributed to building a safe community. One of the facilitators announced to the group that she had discovered she was usually too distracted by her phone during the working sessions, so she was going to put her Cthulhu "Pop! doll" on the phone so she would see him as a reminder. This set a precedent of handling potential pitfalls with humorous-and public-strategies, which were taken up by others. People would, for example, announce goals, and allow the group to set a punishment should the goal not be met (which resulted in entertaining and motivating challenges, such as one of the facilitators dying her hair blue).

\section{It Can Function as a Support Group When Needed}

Having "it's okay" as a starting-point made it possible for the group to also develop an "it's going to be okay" atmosphere. Participants brought up issues and worries that were heavier than simply not meeting hourly goals. Space was found in the conversation for more challenging topics, such as performance anxiety, imposter syndrome, and difficult relationships with supervisors or teachers. These issues were never ignored when brought up by participants, and other participants would immediately jump in to help. Thus the group created a space where members could vent or ask for help. In the focus group, one of the members noted that this practice was very similar to a support group. For example, when faced with a fellow student who was dealing with a particular issue, participants often discussed similar experiences or suggested tactics that had worked for them in the past. Not only did participants make each other feel safe enough to talk about things not going right, they also took an active role in trying to make things go better. Pep talks were regularly given-by anyone, to anyone who needed it-in the brief or debrief.

\section{Safely Assuming the Role of the Host}

Pep talks were also given by facilitators before meetings if the hosts were feeling uncertain about their duties. This feeling of safety for the hosts was important, as it gave them space and comfort to put their own twist on the hosting role: one host led the group in some yoga stretches during the break; another host determined that we were all spending too much time inside and thus decreed that all attendees would take a selfie in an outdoor setting and present it to the group after the break; another host got her cat involved; keeping with the animal theme, another took us outside to show us her neighbor's goat (who had been impeding her progress by bleating loudly), etc. These personal twists were the topics of subsequent conversations in the group, indicating that they too contributed to communitybuilding. 
Safety and Community Foster Critical Reflection

The focus-group students frontloaded the "support group" quality and the feeling of safety that was facilitated for both the role of host and that of attendee as conditional for participants' critical reflection on their own writing process. It was felt by all that the "it's okay" culture opened the door to participants openly examining their own writing habits and, if needed, asking others for help with that critical reflection.

Our findings point to the brief and debrief as spaces where community-building and feelings of safety could be fostered. The safety of the community, in turn, appeared to enable the brief and debrief to serve as places where students could, in a cooperative way, help each other reflect critically on their own and each others' goals and strategies for managing the writing process. The reporting of goals in the brief was done for accountability and support, but we discovered that it can also be used to address affective factors or to help each other think critically about the writing process. For example, attendees shared experiences vis-àvis their respective writing processes, listing, among other things, how they defined and attempted to attain productivity, what common distractions were, and how they approached a wide range of unexpected problems. The LSem students, being third-year students who have dealt with a number of written assignments for various courses, had a latent awareness of their individual writing processes when the writers' group started, but this metacognitive awareness solidified and became more explicit through discussions with their peers. The writers' group functions as a safe environment for experimenting with and reflecting on different approaches to, and aspects of, writing. Hence, the LSem students were able to critically reflect on choices, and develop strategies related to their writing (habits) while they were in the process of writing the most extensive research paper of their academic careers thus far.

With a retrospective look at the intervention, we observe that the safe community and critical reflection worked in a virtuous circle: students felt safe enough with each other to reflect in a critical way on their vulnerabilities, solidifying the community, which made students feel safe, fostering more critical reflection.

\section{Recommendations for Implementation}

Based on our analyses and the enthusiastic response from participants, we would recommend this model as a guideline for the implementation of online writers' groups in other academic environments, both inside and outside of the Covid-19 context. The most important aspects we found from this intervention for new facilitators of online writers' groups can be summarized by the following recommendations:

\section{- Be disciplined but flexible}

The structure should be disciplined, but as mentioned, discipline must be mediated by built-in flexibility. Please consult Appendix for a visual representation of the structure we followed (based on Murray and Newton's [2009] "structured retreat").

- Rotate the host roll

Host rotation will help flatten the hierarchy and increase community-building. 
- Safety first

Use the flexibility and flat hierarchy to foster an "it's okay" culture in which all participants feel safe and empowered in their writing process-even in their failures.

- Leave room for both critical reflection and emotional support Make sure there is a healthy balance between (group) critical reflection on participants' writing process and support group dynamics.

- Allow for play

In our writers' group, we observed that leaving space for playfulness and humor was crucial to building a safe, hierarchically flat community. This aspect may be context-specific, however, and other groups might find that a more serious approach works better for them.

\section{Concluding Remarks: Stay Safe}

The "stay safe" salutation that seems to have become so commonplace during these Covid times is a good reminder for those who seek to develop themselves as writers via online writers' groups, as well as for those who aim to facilitate others in their development. The student members of our group foregrounded emotional safety as one of the most important factors contributing to their perceived benefits of the group, and as that which stimulated regular attendance of meetings. This feeling of safety helped establish a supportive community, opening up a space where writers felt safe to not only critically reflect on their own writing processes, but to also help their peers critically reflect on theirs, and thus develop as writers by making positive changes to their writing processes.

\section{Acknowledgements}

We would like to extend our gratitude to the students, colleagues and friends who participated in the writing sessions during the Covid-19 lockdown March-June 2020. In spite of everything, it was productive. It was fun.

\section{References}

Aitchison, C., \& Guerin, C. (2014). Writing groups for doctoral education and beyond: Innovations in practice and theory. Routledge.

Badenhorst, C., Pickett, S., and Hoben, J. (2019). Writing wild: Writing partnerships that fly. In N. Simmons \& A. Singh (Eds.), Critical collaborative communities: Academic writing partnerships, groups, and retreats (pp. 121-135). Brill Sense.

Cottrell, S. (2017). Critical thinking skills: Effective analysis, argument and reflection. Palgrave Macmillan.

Elbow, P. (1998). Writing without teachers. Oxford University Press.

Erdogan, F. (2020). The relationship between prospective middle school mathematics teachers' critical thinking skills and reflective thinking skills. Participatory Educational Research, 7, 220-241.

Gere, A. R. (1987). Writing groups. Southern Illinois University Press.

Ghanizadey, A. (2017). The interplay between reflective thinking, critical thinking, selfmonitoring, and academic achievement in higher education. Higher Education, 74, 101-114. 
Hennekam, S., Macarthur, S., Bennett, D., Hope, C., \& Goh, T. (2019). Women composers' use of online communities of practice to build and support their careers. Personnel Review, 49, 215-230. https://doi.org/10.1108/PR-02-2018-0059

Haas, S. (2009). Writers' groups for MA ESOL students: Collaboratively constructing a model of the writing process. ELTED Journal 12, 23-30.

Haas, S. (2014). Pick-n-mix: A typology of writers groups in use. In C. Aitchison \& C. Guerin (Eds.), Writing groups for doctoral education and beyond: Innovations in practice and theory (pp. 30-47). Routledge.

Haas, S., \& Kobayashi, S. (2014). Writer development made accessible! Brookes eJournal of Learning and Teaching, 6(2).

Haas, S. (2020). Writer development course 2019-2020. Doctoral Schools, Ghent University. https://www.ugent.be/doctoralschools/en/doctoraltraining/courses/transferables kills/all/writer-development-course.htm

Ji, H., Sui, Y., \& Suo, L. (2017). Understanding innovation mechanism through the lens of communities of practice (COP). Technological Forecasting and Social Change, 118, 205-212. https://doi.org/10.1016/j.techfore.2017.02.021

Kember, D., McKay, J., Sinclair, K., \& Wong, K.Y. (2008). A four-category scheme for coding and assessing the level of reflection in written work. Assessment \& Evaluation in Higher Education, 33, 369-379.

Kornhaber, R., Cross, M., Betihavas, V., \& Bridgman, H. (2016). The benefits and challenges of academic writing retreats: An integrative review. Higher Education Research \& Development, 35, 1210-1227. https://doi.org/10.1080/07294360.2016.1144572

Mewburn, I., Osborne, L., \& Galdwell, G. (2014). Shut up \& write! Some surprising uses of cafés and crowds in doctoral writing. In C. Aitchison \& C. Guerin (Eds.), Writing groups for doctoral education and beyond: Innovations in practice and theory (pp. 218232). Routledge.

Murray, R. (2015). Writing in social spaces: A social processes approach to academic writing. Routledge.

Murray, R., \& Newton, M. (2009). Writing retreat as structured intervention: Margin or mainstream? Higher Education Research \& Development, 28, 541-553. https:// doi.org/10.1080/07294360903154126

O'Dwyer, S. T., McDonough, S. L., Jefferson, R., Goff, J. A., \& Redman-MacLaren, M. (2017). Writing groups in the digital age: A case study analysis of shut up \& write Tuesdays. In A. Esposito (Ed.), Research 2.0 and the impact of digital technologies on scholarly inquiry (pp. 249-269). IGI Global.

Rademaekers, J.K., \& Detweiler, L. (2019). Performing critical thinking in written language: Defining critical thinking from the assessor's veiw. Double Helix 7, 1-19.

Thesen, L. (2014). If they're not laughing, watch out! Emotion and risk in postgraduate writers' circles. In C. Aitchison \& C. Guerin (Eds.), Writing groups for doctoral education and beyond: Innovations in practice and theory (pp.162-176). Routledge. 


\section{Appendix}

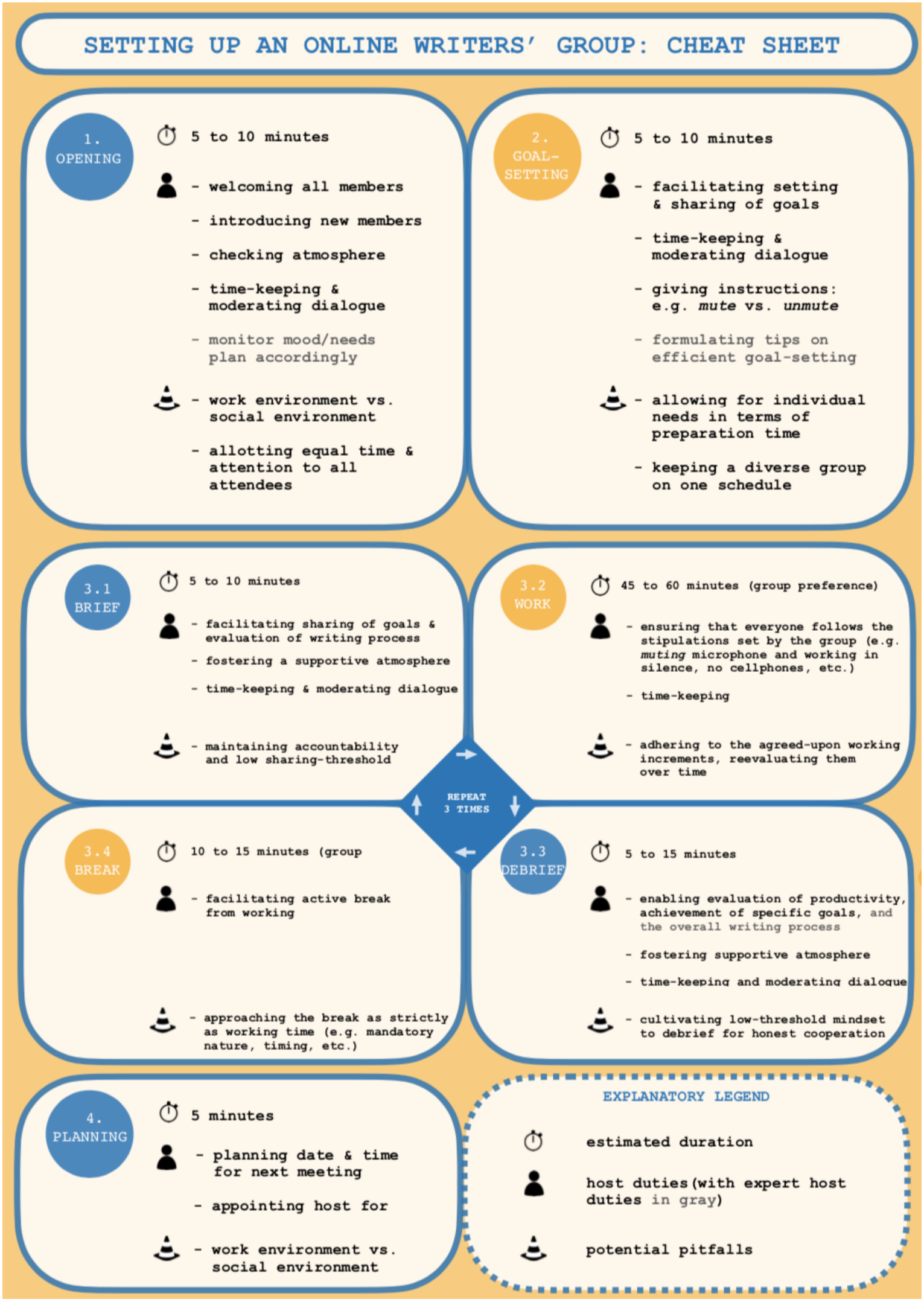

